\title{
Bacteria-Derived Recombinant Human ANGPTL8/Betatrophin Significantly Increases the Level of Triglyceride
}

\author{
Fangfang $\mathrm{Xu}^{1,2}$ (D) Yuqing $\mathrm{Chen}^{3} \cdot \mathrm{Nan} \mathrm{Wang}^{2} \cdot$ Kai Sun ${ }^{3}$
}

Published online: 30 March 2019

(c) The Author(s) 2019

\begin{abstract}
ANGPTL8/Betatrophin has been implicated in the regulation of both glucose and triglyceride metabolism. However, its role in regulating glucose metabolism by promoting $\beta$ cell proliferation remains controversial, and its physiological functions and molecular targets are largely unknown. Hence, it is of great importance to make recombinant protein and test its effects on $\beta$ cell mass directly. In this study, the mature form gene of human ANGPTL8/betatrophin was obtained through chemical synthesis on to the vector pUCE, and the fusion protein was expressed in the Transetta (DE3)/pEASY-E2-betatrophin strain. The inclusion bodies were solubilized in urea and purified by Ni-NTA affinity chromatography. The yield of purified ANGPTL8/betatrophin was approximately $20 \mathrm{mg}$ per liter of culture medium. In vitro studies revealed that the recombinant ANGPTL8/betatrophin had no proliferation effect on MIN6 cells but promoted TG levels in HepG2 cells. This method to generate bioactive ANGPTL8/betatrophin is a simple, practical and user-friendly protocol.
\end{abstract}

Keywords ANGPTL8/betatrophin · Recombinant expression · Beta cell proliferation · Triglyceride level

\section{Introduction}

Type 2 diabetes mellitus (T2D), characterized by hyperglycemia, results from dysfunctional carbohydrate metabolism that is caused by a relative deficiency of insulin. T2D has become a major health burden, with current prevalence estimated at 425 million and predicted to reach 629 million by 2045 worldwide [1]. Hence, The identification of novel molecules regulating glucose metabolism might provide new therapeutic targets for the treatment of T2D.

Betatrophin, also known as TD26 [2], refeeding induced fat and liver (RIFL) [3], lipoprotein lipase inhibition (lipasin) [4], and atypical angiopoietin-like protein 8 (ANGPTL8) [5], is primarily secreted by the human liver and white adipose tissue under the conditions of insulin resistance [6];

Fangfang Xu

xffxff0924@163.com

1 Department of Research and Discipline Development, Henan Provincial People's Hospital, No. 7 Weiwu Road, Jinshui District, Zhengzhou, Henan 450003, China

2 Biotechnology Research Institute, Chinese Academy of Agricultural Sciences, Beijing 100081, China

3 Department of Hematology, Henan Provincial People's Hospital, Zhengzhou 450003, China here we refer to it as ANGPTL8/betatrophin. ANGPTL8/ betatrophin has been implicated in both glucose and triglyceride metabolism [7, 8]. It has been reported to improve glucose tolerance in mice by promoting $\beta$ cell proliferation in response to insulin resistance [6,9], which has raised hopes for seeking a novel therapeutic target to treat diabetes. However, subsequent studies have led to conflicting claims about its physiological roles in promoting $\beta$ cell proliferation $[10$, 11]. These findings suggested that ANGPTL8/betatrophin was involved in triglyceride metabolism rather than glucose homeostasis [12]. Systematic review and meta-analysis indicated a preference for association between betatrophin and T2DM [13-15]. Hence, the role of ANGPTL8/betatrophin in $\beta$ cell proliferation and glucose metabolism seems controversial. We do not yet know the mechanism of betatrophin action, which points to the importance of producing recombinant betatrophin and testing it directly for the effects on $\beta$ cell mass [6]. In this study, recombinant ANGPTL8/betatrophin was successfully expressed in Escherichia coli, and the biological activity of the purified protein was tested in vitro. 


\section{Materials and Methods}

\subsection{Vectors, Strains, Cell Lines and Reagents}

Escherichia coli cloning vector pEASY-T1, expression vector pEASY-E2, competent cells Trans1-T1 and Transetta (DE3) were purchased from TransGen Biotech (Beijing, China). The whole gene of the mature form of ANGPTL8/ betatrophin was synthesized by Inovogen Tech. Co. (Beijing, China) into pUCE plasmids. The MIN6 cell line was obtained from iCell Bioscience Inc. (Shanghai, China). The HepG2 cell line was donated by Dr. Hu Xiaoyuan (Biotechnology Research Institute, Chinese Academy of Agricultural Sciences). The triglyceride enzyme assay kit was purchased from Applygen Technologies Inc. (Beijing, China). All other chemicals and reagents, unless otherwise stated, were purchased from Beijing Solarbio Science and Technology Co., Ltd. (Beijing, China).

\subsection{Construction of the Expression Plasmid, pEASY-E2-Betatrophin}

Full-length mature form DNA of human ANGPTL8/betatrophin (555 bp) was obtained through PCR with pUCE-betatrophin plasmid as templates. The gene-specific primer pair was list as follows: Betatrophin-F: 5'-ATGGCTCCAATG GGTGGTCCAGAAT-3', Betatrophin-R: 5'-CCTAGGTTA ATGGTGATGGTGATGG-3'. It was synthesized by Sangon Biotech Co., Ltd. (Shanghai, China). The PCR product was separated by $1 \%$ agarose gel electrophoresis and purified by a gel extraction kit. Subsequently, it was cloned into the pEASY-E2 expression vector by TA cloning which was then transformed into $E$. coli trans1-T1 clone strains. The constructed plasmid pEASY-E2-betatrophin was abstracted and used as PCR templates. The PCR products were confirmed by gel electrophoresis as well as DNA sequencing. Then the plasmid was again transformed into E. coli Transetta (DE3) strain. Transetta (DE3)/pEASY-E2-betatrophin was used as engineering strain for the following work.

\subsection{Expression and Confirmation of Recombinant ANGPTL8/Betatrophin by SDS-PAGE and Western Blotting}

The Transetta (DE3)/pEASY-E2-betatrophin strain was cultured in $10 \mathrm{~mL}$ Luria-Bertani (LB) medium with $50 \mathrm{mg} / \mathrm{L}$ ampicillin at $37{ }^{\circ} \mathrm{C}$ to an $\mathrm{OD}_{600}=0.6-0.8$. One $\mathrm{mmol} / \mathrm{L}$ isopropy- $\beta$-D-thiogalactoside (IPTG) was added into the culture and incubated for approximately 6-8 h. Cells were harvested and ultrasonicated in phosphate buffer saline (PBS) (137 mmol/L NaCl, $2.7 \mathrm{mmol} / \mathrm{L} \mathrm{KCl,} 10 \mathrm{mmol} / \mathrm{L} \mathrm{Na}_{2} \mathrm{HPO}_{4}$, $\left.2 \mathrm{mmol} / \mathrm{L} \mathrm{KH}_{2} \mathrm{PO}_{4}, \mathrm{pH} 7.4\right)$. After centrifugation at $4{ }^{\circ} \mathrm{C}$, $12,000 \mathrm{rpm}$ for $10 \mathrm{~min}$, both supernatant and insoluble fractions were collected and separated by sodium dodecyl sulfate-polyacrylamide gel electrophoresis (SDS-PAGE) with coomassie blue R-250 staining. The proteins separated on the SDS-PAGE gels were transferred onto a polyvinylidene fluoride (PVDF) membrane (Sigma-Aldrich, United States). Subsequently, western blotting was performed using goat anti-human betatrophin polyclonal antibody (A00528-11100, Aviscera Bioscience, Inc., Santa Clara, USA) in 1:5000 dilution and rabbit anti-goat immunoglobulin G-alkaline phosphatase (IgG-AP, ab6742, Abcam Ltd., Cambridge, United Kingdom) in 1:3000 dilution visualized with 5-bromo-4-chloro-3-indolyl phosphatase/nitroblue tetrazolium chloride (BCIP/NBT) solution.

\subsection{Optimizing the Expression Conditions of ANGPTL8/Betatrophin}

The optimal conditions for ANGPTL8/betatrophin expression, including IPTG concentration, induction time and temperature, were determined prior to large-scale production. The recombinant $E$. coli strain Transetta (DE3) carrying the pEASY-E2-betatrophin construct was induced with $0,0.1$, $0.3,0.5,0.8,1.0$, or $1.2 \mathrm{mmol} / \mathrm{L}$ IPTG at $200 \mathrm{rpm}, 37^{\circ} \mathrm{C}$, for $8 \mathrm{~h}$. The optimum IPTG concentration was determined using SDS-PAGE analysis visualized by coomassie blue R-250 staining. The Transetta (DE3)/pEASY-E2-betatrophin strain was then induced using the determined optimum concentration of IPTG at $200 \mathrm{rpm}, 37^{\circ} \mathrm{C}$, for $0,1,2,4,6,8,10$, or 12 h. Finally, the Transetta (DE3)/pEASY-E2-betatrophin strain was induced at $200 \mathrm{rpm}$ using the optimum IPTG for an optimal time at 16,30 , or $37^{\circ} \mathrm{C}$.

\subsection{Large Scale Production and Purification of Recombinant ANGPTL8/Betatrophin}

The engineered bacterial strain Transetta (DE3)/pEASYE2-betatrophin was cultured in $500 \mathrm{~mL} \mathrm{LB}$ medium with $50 \mathrm{mg} / \mathrm{L}$ ampicillin at $37{ }^{\circ} \mathrm{C}$. When the $\mathrm{OD}_{600}$ was reached $0.6-0.8,0.3 \mathrm{mmol} / \mathrm{L}$ IPTG was added into the culture and incubated for $8 \mathrm{~h}$. Cells were harvested and disrupted by ultrasonication after resuspension in PBS buffer. Following centrifugation at $15,000 \mathrm{rpm}$ for $30 \mathrm{~min}$ at $4{ }^{\circ} \mathrm{C}$, inclusion bodies were collected and dissolved in $8 \mathrm{~mol} / \mathrm{L}$ urea solution. Purification was performed using nickel-nitrilotriacetic acid (Ni-NTA) bind resin in a His-tagged affinity chromatography column (Qiagen, Hilden, Germany). Fusion proteins were then subjected to gradient dialysis for renaturation. The dry powder of the purified betatrophin-His fusion protein obtained from lyophilization was kept at $4{ }^{\circ} \mathrm{C}$ for further use. 


\subsection{HPLC Analysis}

High-performance liquid chromatography (HPLC) assay was performed by use of LC-2010A/C chromatographic instrument (Shimadzu, Japan) with a C18 column $(250 \times 4.6 \mathrm{~mm}$, I.D.S-5 $\mu \mathrm{m}, 12 \mathrm{~nm}$, YMC-Pack ODS-A, Germany). The mobile phase was acetonitrile/water $(90: 10$, v/v) with $0.1 \%$ trifluoroacetic acid (TFA). The gradient assay was performed at ambient temperature at a flow rate of $1.0 \mathrm{~mL} / \mathrm{min}$ and lasted for $20 \mathrm{~min}$. A commercial betatrophin standard (00528-01-100, $100 \mu \mathrm{g}$, Aviscera Bioscience, USA) and a blank of PBS ( $\mathrm{pH} 7.4$ ) were used as controls. All samples were detected by measurements at $280 \mathrm{~nm}$.

\subsection{Cell Proliferation Assay}

To determine the promoting effect of $E$. coli-derived betatrophin on cell proliferation, a mouse pancreatic $\beta$ cell line, MIN6, was used to evaluate the effect of recombinant betatrophin (r-betatrophin) on cell proliferation. Cells were cultured in Roswell Park Memorial Institute (RPMI) 1640 medium (Gibco, USA) with $10 \%$ (v/v) fetal bovine serum (FBS), $2 \mathrm{mmol} / \mathrm{L}$ L-glutamine, $50 \mu \mathrm{mol} / \mathrm{L} 2$-mercaptoethanol, $100 \mathrm{U} / \mathrm{mL}$ penicillin and $0.1 \mathrm{mg} / \mathrm{mL}$ streptomycin at a high $(95 \%)$ relative humidity $(\mathrm{RH})$ and $5 \% \mathrm{CO}_{2}$ at $37^{\circ} \mathrm{C}$. At a density of $3 \times 10^{4}$ cells per well, MIN6 cells were seeded into a flat-bottomed 96-well microtiter plate. Twentyfour hours later, cells were washed twice with Dulbecco's phosphate buffered saline (DPBS, Corning, USA). Subsequently, purified r-betatrophin at a concentration of 1.5, 5, 10,15 , or $25 \mu \mathrm{mol} / \mathrm{L}$ was added into the culture medium $(\mathrm{n}=6)$. After 48 -h incubation, $10 \mu \mathrm{L}$ Cell Counting Kit-8 (CCK-8, Dojindo, Japan) was added to each well according to the manufacture's instructions. CCK- 8 kit utilizes the water-soluble tetrazolium salt-WST ${ }^{\circledR}-8$ (2-(2-methoxy4-nitrophenyl)-3-(4-nitrophenyl)-5-(2, 4-disulfobenzene)$2 \mathrm{H}$-tetrazole mono-sodium salt) developed by Dojindo. The orange yellow formazan dye produced by the oxidation and reduction of $\mathrm{WST}^{\circledR}-8$ by intracellular dehydrogenase can be dissolved in culture medium, and the amount of formazan produced is proportional to the number of living cells. So cultures were incubated for an additional 1-4 h. Finally, cell viability of all samples was analyzed in a microplate reader at $450 \mathrm{~nm}$ wavelength. The experiment was repeated twice for reproducibility.

\subsection{Measurement the Concentration of Triglyceride}

HepG 2 cells were treated with $200 \mathrm{nmol} / \mathrm{L}$ r-betatrophin for $48 \mathrm{~h}(\mathrm{n}=3)$. The cultures were then collected for determination of the triglyceride (TG) concentrations using a Triglyceride Quantification Assay Kit (E1003, Applygen Technologies Inc., China) according to the manufacturer's instructions. The principle of the kit is as follows: (1) triglyceride was decomposed into glycerol by lipase; (2) glycerol was phosphorylated to glycerol-3-phosphate by glycerol kinase; (3) glycerol 3-phosphate was oxidized by glycerol phosphate oxidase to produce hydrogen peroxide and under the action of peroxidase, the chromogenic substrate was converted to benzoquinone. The color would be stabilized within 60 min after the reaction was equilibrated. The optical density value was proportional to the triglyceride concentration and the value of each tube was measured at $550 \mathrm{~nm}$ wavelength. Drew a standard curve and then calculated the triglyceride concentration. The experiment was repeated twice for reproducibility.

\section{Results}

\subsection{Construction of Expression Plasmid pEASY-E2-Betatrophin}

Full-length DNA of mature form of human ANGPTL8/ betatrophin (177 amino acids) was synthesized into vector pUCE. Using pUCE-betatrophin plasmid as the PCR template, 555 bp gene of fusion protein betatrophin- $6 \times$ Histag was amplified with gene-specific primers. PCR products were then inserted into the purchased cloning vector pEASY-T1 by TA cloning and transformed into Trans1-T1. After PCR determination and sequence analysis, the gene of the fusion protein was amplified again using pEASYT1-betatrophin plasmid as the PCR template. The products were purified and again inserted into the expression vector pEASY-E2 by TA cloning and transformed into Transetta (DE3) strain. The establishment process and the result of PCR determination using Transetta (DE3)/pEASY-E2-betatrophin clones as templates are shown in Fig. 1.

\subsection{Analyzing the Expression of Recombinant Betatrophin}

The E. coli Transetta (DE3)/betatrophin strain was induced using IPTG for expression. Samples of supernatant and precipitate were obtained by centrifugation after collection and ultrasonication. The results of SDS-PAGE and western blotting analysis showed that r-betatrophin was rarely detected in the supernatant and highly expressed in the insoluble form of inclusion bodies (Fig. 2a, b). It indicated that the molecular weight (MW) of r-betatrophin was less than $25 \mathrm{kDa}$, which is consistent with the theoretically estimated MW of $22 \mathrm{kDa}$. It also indicated that the fusion protein was not degraded. 
Fig. 1 Construction and validation of the expression vector pEASY-E2-betatrophin. a Protein structure of ANGPTL8/ betatrophin. $S P$ signal peptide. b Construction and identification of the expression vector pEASY-E2-betatrophin. Lane 1: DNA Marker; Lanes 2-3: PCR products using Transetta (DE3)/pEASY-E2-betatrophin clones as PCR templates; Lane 4: positive control, using PUCEbetatrophin plasmid as the PCR template
A
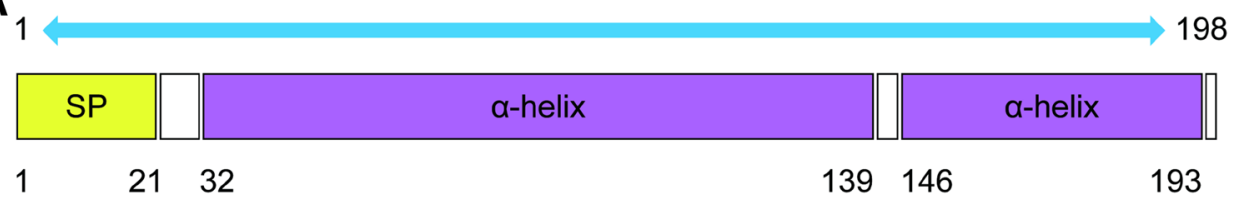

B
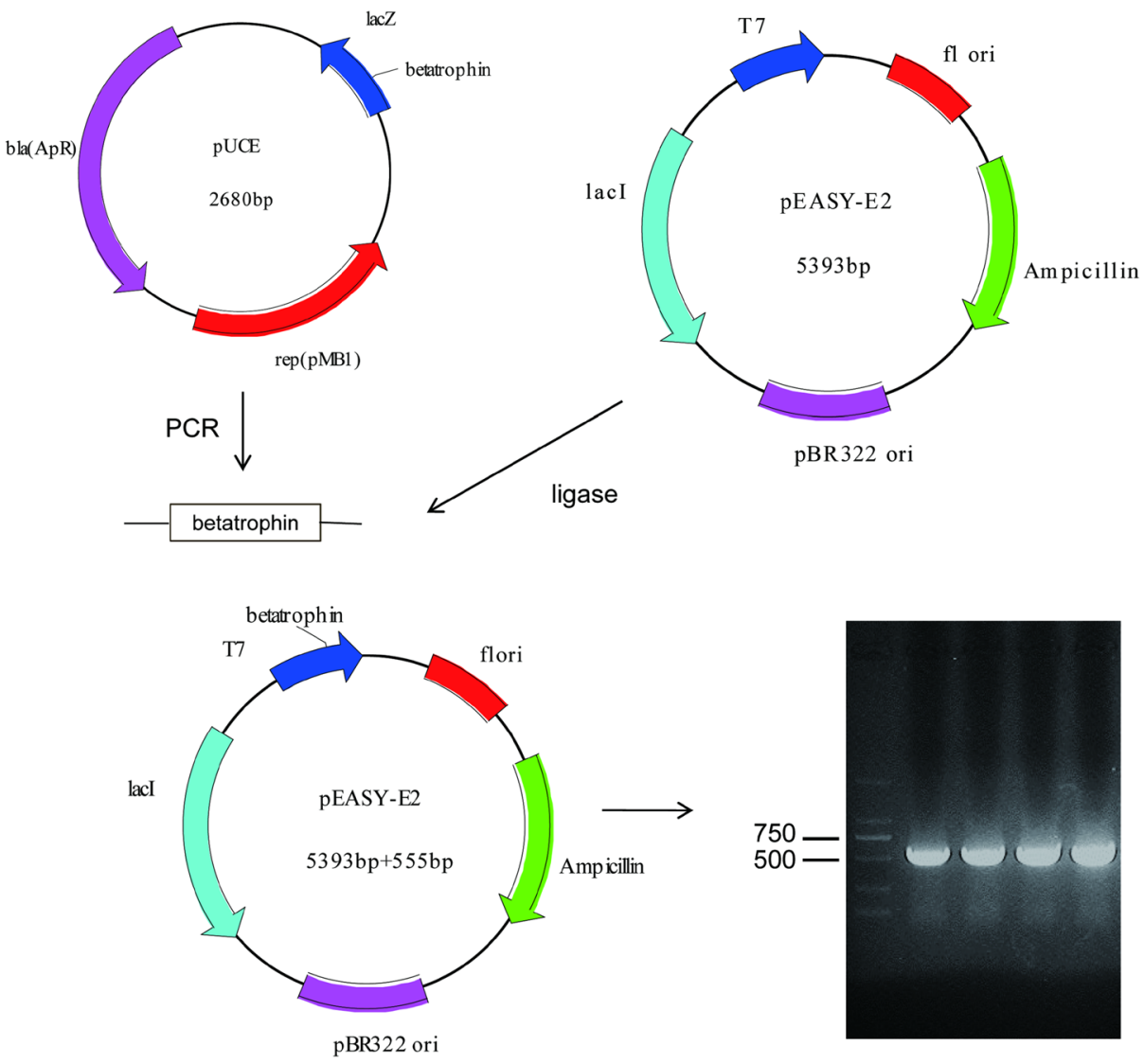

\subsection{Optimization of Recombinant Betatrophin Expression and Purification}

To maximize the output of r-betatrophin products, different concentrations of IPTG $(0.1,0.3,0.5,0.8,1.0$, or $1.2 \mathrm{mmol} / \mathrm{L})$, culture time $(1,2,4,6,8,10$, or $12 \mathrm{~h})$ and temperatures $\left(16,30\right.$, or $\left.37^{\circ} \mathrm{C}\right)$ were employed. The SDS-PAGE analysis showed that the optimal concentration of IPTG was $0.3 \mathrm{mmol} / \mathrm{L}$, and the optimal induction time was $8 \mathrm{~h}$ (Fig. 3a, b). However, even though the culture temperature was as low as $16{ }^{\circ} \mathrm{C}$, little recombinant protein was present in the supernatant. Based on this, $30{ }^{\circ} \mathrm{C}$ was selected as the optimal culture temperature for the rapid growth and metabolic activity of $E$. coli.

Transetta (DE3)/pEASY-E2-betatrophin was induced under optimal expression conditions in order to obtain abundant r-betatrophin. After centrifugation following PBS ( $\mathrm{pH}$ 8.0) resuspension, the inclusion bodies were solubilized using $8 \mathrm{~mol} / \mathrm{L}$ urea in combination with ultrasonication when necessary. Subsequently, the soluble expression products were purified by Ni-NTA affinity chromatography. The fusion protein was then renatured by gradient dialysis to gradually remove urea via a graded series of PBS buffer (pH 7.4). After lyophilization, dry powder form of purified betatrophin was obtained. SDS-PAGE analysis demonstrated that $r$-betatrophin of high quality was successfully obtained (Fig. 4a). HPLC analysis indicated that the purity of r-betatrophin was approximately $100 \%$ (Fig. 4b). The retention time of the peak was $15.727 \mathrm{~min}$, consistent with $15.733 \mathrm{~min}$ of a commercial standard, as shown in Fig. 4b. The yield of purified recombinant betatrophin was approximately $20 \mathrm{mg}$ per liter of culture medium.

\subsection{Bioactivity Assay of Recombinant Betatrophin}

MIN6 cell line was used to detect the proliferation effect of betatrophin. In vitro, r-betatrophin was directly added to the cell culture at different concentrations, with DPBS-treated 


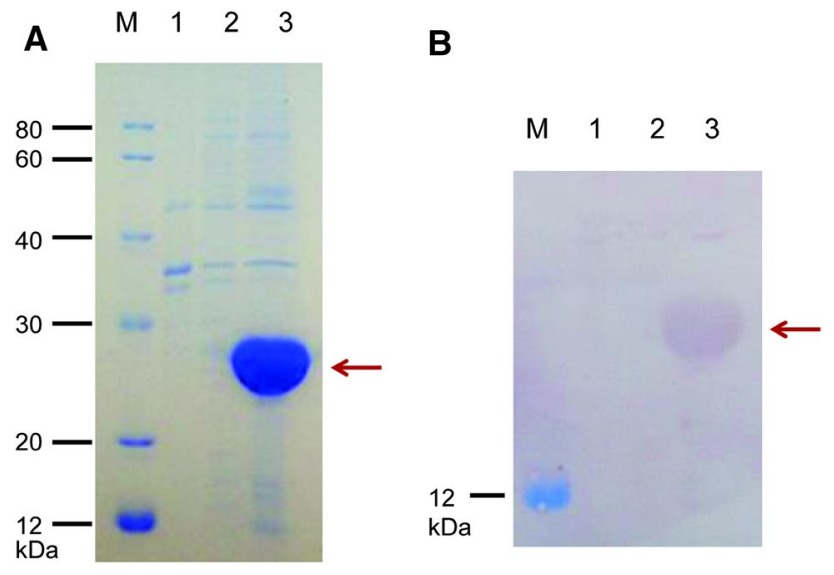

Fig. 2 SDS-PAGE and western blot analysis of betatrophin. a Representative result of SDS-PAGE. b Representative result of western blot. M: protein marker; Lane 1: Transetta (DE3)/pEASY-E2-betatrophin strain without IPTG induction; Lanes 2 and 3: the supernatant and precipitate of Transetta (DE3)/pEASY-E2-betatrophin strain obtained from cell lysis and centrifugation following IPTG induction. Betatrophin is indicated by a red arrow (Color figure online)
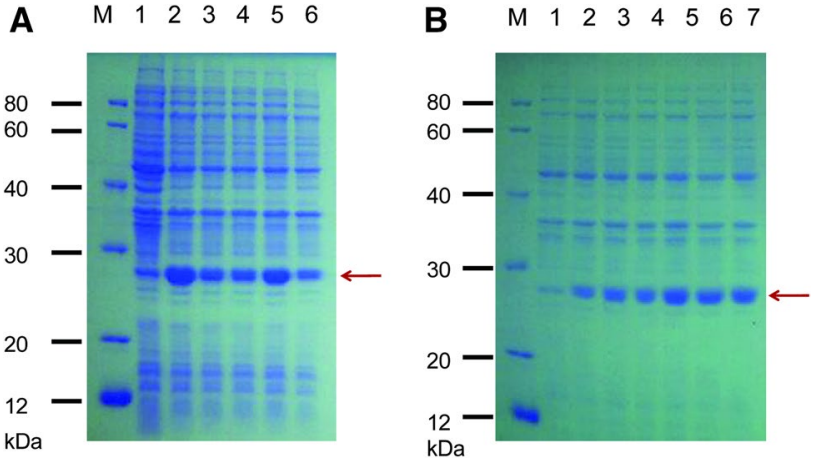

Fig. 3 Optimizing prokaryotic expression conditions of betatrophin. a SDS-PAGE analysis of optimum IPTG concentration. M: Protein marker; Lanes 1-6: Transetta (DE3)/pEASY-E2-betatrophin strain induced by $0.1,0.3,0.5,0.8,1.0$, and $1.2 \mathrm{mmol} / \mathrm{L}$ IPTG, respectively, at $30{ }^{\circ} \mathrm{C}, 200 \mathrm{rpm}$ for $8 \mathrm{~h}$. b SDS-PAGE analysis of optimum induction time. M: Protein marker; Lanes 1-7: Transetta (DE3)/ pEASY-E2-betatrophin strain induced for $1,2,4,6,8,10$, and $12 \mathrm{~h}$, respectively, with optimum IPTG concentration at $30{ }^{\circ} \mathrm{C}, 200 \mathrm{rpm}$. Recombinant betatrophin protein is indicated by a red arrow (Color figure online)

cells as the negative control. After incubation for $48 \mathrm{~h}, 10 \mu \mathrm{L}$ CCK-8 solution were added into each well. One to four hours later, cell density was measured at $450 \mathrm{~nm}$ wavelength. The results demonstrated that compared with DPBS-treated controls, there was no significant difference in cell density for r-betatrophin-treated cells, indicating that betatrophin likely has no proliferation effect on $\beta$ cells (Fig. 5a).

HepG2 cell line was used to detect the effect of betatrophin on TG metabolism. HepG2 cells were treated with
$200 \mathrm{nmol} / \mathrm{L}$ r-betatrophin for $48 \mathrm{~h}$. The cell cultures were then collected to measure TG levels. Our results showed that the concentrations of TG in the culture of r-betatrophintreated cells were significantly higher than that of DPBStreated cells $(P=0.002)$ (Fig. 5b).

\section{Discussion}

ANGPTL8/Betatrophin is a newly characterized hormone which is primarily secreted by human liver and white adipose tissue. Till now, the function and mechanism of which is still unknown. It was initially reported to be involved in the control of $\beta$ cell proliferation in mice response to insulin resistance [6]. Also, transplantation of adipose-derived mesenchymal stem cells expressing betatrophin induced human $\beta$ cell proliferation [9]. However, subsequent studies revealed that ANGPTL8/betatrophin did not control pancreatic $\beta$ cell expansion in Angptl8 gene knockout/overexpression mice or mice transplanted with human $\beta$ cells $[10$, $11,16]$. Moreover, overexpression of ANGPTL8 resulted in doubled plasma TG levels [10]. Multiple studies have indicated that betatrophin was involved in TG metabolism [4, 12, $17,18]$. Nevertheless, several groups independently found that the circulating betatrophin concentrations were correlated with insulin resistance or type 2 diabetes [14, 19-22]. Recently it has been reported to potentially be involved in hypertension [23], polycystic ovary syndrome [24-26], and urinary albumin excretion and renal function [27], which indicates it is a pleiotropic hormone.

The function of betatrophin is not clear and in some cases controversial. To understand the function of betatrophin in detail, it is necessary to make recombinant protein with high purity in adequate quantities.

In this study, we attempted to simplify the production procedure of recombinant betatrophin with high yield. The recombinant ANGPTL8/betatrophin was highly expressed in Transetta (DE3) cells, and the optimized expression conditions were induced with $0.3 \mathrm{mmol} / \mathrm{L}$ IPTG at $30{ }^{\circ} \mathrm{C}$ for $8 \mathrm{~h}$. Ni-NTA His-bind Resin affinity chromatography was used for purification and HPLC for purity measurement. The entire production procedure is easy to implement with no need for specialized equipment. In vitro studies demonstrated that ANGPTL8/betatrophin did not promote MIN6 cell proliferation, but increased triglyceride levels in the culture of HepG2 cells, which supports the possibility that inhibition of ANGPTL8/Betatrophin represents a therapeutic strategy for hypertriglyceridemia [10]. Although the results of the biological assays were partially consistent with previous studies and showed that the recombinant betatrophin derived from bacteria was bioactive, the mechanism of it in the regulation of glucose metabolism is still unclear. As the dry lab revealed the 

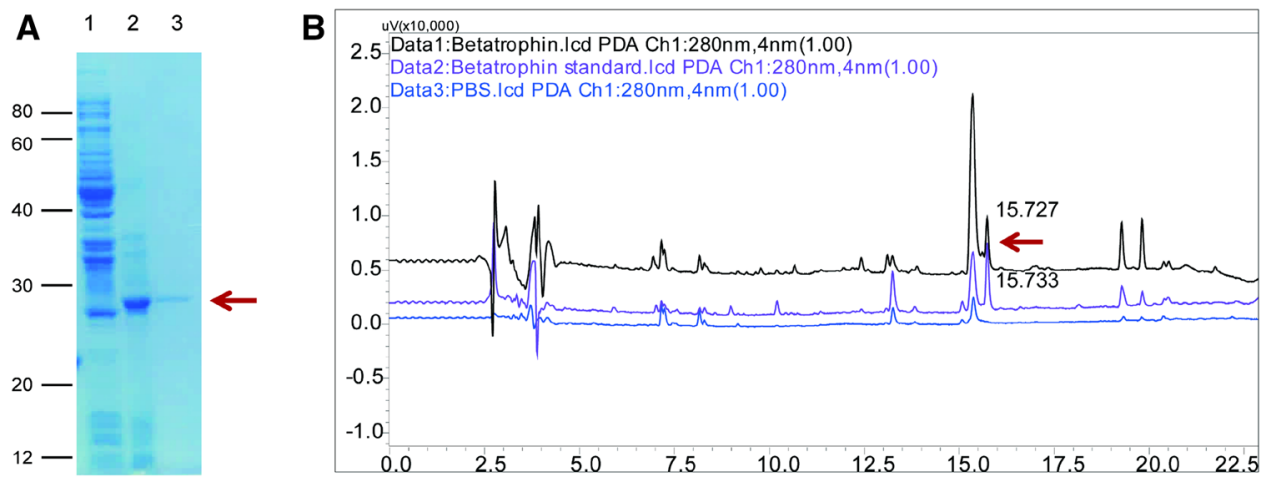

Fig. 4 Purification analysis of r-betatrophin. a SDS-PAGE analysis of r-betatrophin with coomassie brilliant blue staining. Lane 1: Transetta (DE3)/pEASY-E2-betatrophin strain without IPTG induction; Lane 2: inclusion bodies form of r-betatrophin; Lane 3: dry powder of puri-
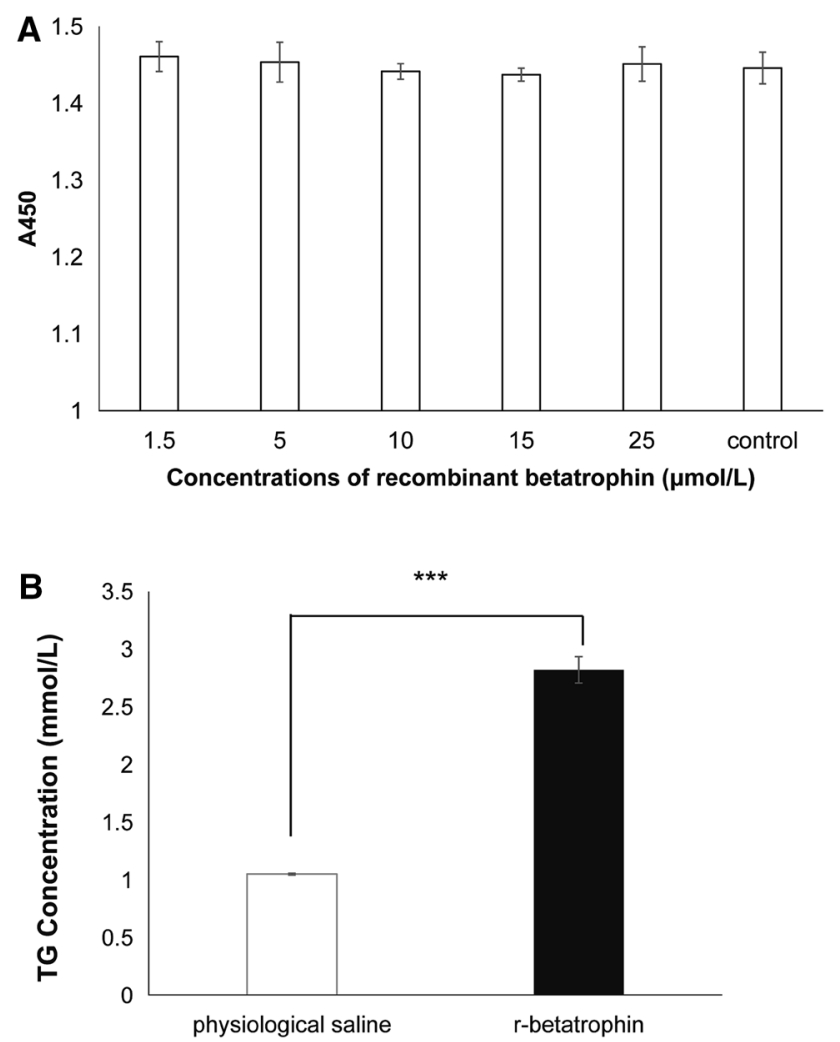

Fig. 5 Biological activity of r-betatrophin. Effect of recombinant betatrophin on the proliferation of MIN6 cells $(n=6)(a)$ and TG matabolism of HepG2 cells $(n=3)(b)$. All experiments were repeated three times and “***" indicates $P<0.001$

regulatory role of ANGPTL8/betatrophin both in glucose and lipid metabolic pathways [28], further wet lab studies on its functional mechanism must be performed, and our procedure for obtaining active recombinant betatrophin in high quantities provides a material basis. fied r-betatrophin reconstructed in PBS (pH 7.4). b HPLC analysis of $\mathrm{r}$-betatrophin. Betatrophin is indicated by a red arrow (Color figure online)

Acknowledgments This work was supported by the postdoctoral research start-up funds of Henan Provincial People's Hospital. We also thank Professor Xiaoyuan Hu from the Chinese Academy of Agricultural Sciences for kindly providing us with the HepG2 cell line.

Authors' Contributions FX conceived the study, analyzed data and wrote the manuscript. YC performed the study, analyzed data and prepared the manuscript. NW designed the experiments, analyzed data and prepared the manuscript. KS prepared the experiments and the manuscript.

\section{Compliance with Ethical Standards}

Conflict of interest The authors declare that they have no conflict of interest.

Ethical Approval This article does not contain any studies with human participants or animals performed by any of the authors.

Open Access This article is distributed under the terms of the Creative Commons Attribution 4.0 International License (http://creativeco mmons.org/licenses/by/4.0/), which permits unrestricted use, distribution, and reproduction in any medium, provided you give appropriate credit to the original author(s) and the source, provide a link to the Creative Commons license, and indicate if changes were made.

\section{References}

1. https://diabetesatlas.org/. Accessed 1 Dec 2017

2. Dong XY, Pang XW, Yu ST et al (2004) Identification of genes differentially expressed in human hepatocellular carcinoma by a modified suppression subtractive hybridization method. Int $\mathbf{J}$ Cancer 112(2):239-248

3. Ren G, Kim JY, Smas CM (2012) Identification of RIFL, a novel adipocyte-enriched insulin target gene with a role in lipid metabolism. Am J Physiol Endocrinol Metab 303(3):E334-E351

4. Ren Z (2012) Lipasin, a novel nutritionally-regulated liverenriched factor that regulates serum triglyceride levels. Biochem Biophys Res Commun 424(4):786-792 
5. Quagliarini F, Wang Y, Kozlitina J et al (2012) Atypical angiopoietin-like protein that regulates ANGPTL3. Proc Natl Acad Sci USA 109(48):19751-19756

6. Yi P, Park JS, Melton DA (2014) Betatrophin: a hormone that controls pancreatic $\beta$ cell proliferation. Islets 153(2):747-758

7. Zhu JZ, Yu CH, Li YM (2014) Betatrophin provides a new insight into diabetes treatment and lipid metabolism. Biomed Rep 2(4):447-451

8. Abu-Farha M, Abubaker J, Tuomilehto J (2017) ANGPTL8 (betatrophin) role in diabetes and metabolic diseases. Diabetes Metab Res Rev 33(8):1-19

9. Sun LL, Liu TJ, Li L et al (2017) Transplantation of betatrophinexpressing adipose-derived mesenchymal stem cells induces $\beta$-cell proliferation in diabetic mice. Int J Mol Med 39(4):936-948

10. Gusarova V, Alexa CA, Na E et al (2014) ANGPTL8 (betatrophin) does not control pancreatic beta cell expansion. Cell 159(3):691-696

11. Cox AR, Lam CJ, Bonnyman CW et al (2015) Angiopoietinlike protein 8 (ANGPTL8)/betatrophin overexpression does not increase beta cell proliferation in mice. Diabetologia 58(7):1523-1531

12. Wang Y, Quagliarini F, Gusarova V et al (2013) Mice lacking ANGPTL8 (betatrophin) manifest disrupted triglyceride metabolism without impaired glucose homeostasis. Proc Natl Acad Sci USA 110(40):16109-16114

13. Li S, Dan L, Ling L et al (2016) Circulating betatrophin in patients with type 2 diabetes: a meta-analysis. J Diabetes Res 1:1-22

14. Song Y, Wu J, Zhang J et al (2016) The relationship between betatrophin levels in blood and T2DM: a systematic review and meta-analysis. Dis Mark 3:1-15

15. Dan L, Li S, He H et al (2017) Increased circulating full-length betatrophin levels in drug-naïve metabolic syndrome. Oncotarget 8(11):17510-17517

16. Jiao Y, Lay JL, Yu M et al (2014) Elevated mouse hepatic betatrophin expression does not increase human $\beta$-cell replication in the transplant setting. Diabetes 63(4):1283-1288

17. Zhang R (2016) The ANGPTL3-4-8 model, a molecular mechanism for triglyceride trafficking. Open Biol 6(4):1-37

18. Zhang Y, Li S, Donelan W et al (2016) Angiopoietin-like protein 8 (betatrophin) is a stress response protein that down-regulates expression of adipocyte triglyceride lipase. Biochim Biophys Acta Mol Cell Biol Lipids 1861(2):130-137
19. Tuhan H, Abacı A, Anık A et al (2016) Circulating betatrophin concentration is negatively correlated with insulin resistance in obese children and adolescents. Diabetes Res Clin Pract 114:37-42

20. Xu J, Lin Y, Zhou H et al (2017) The correlation between circulating betatrophin and insulin resistance in general population: a meta-analysis. Horm Metab Res 49(10):760-771

21. Wang YY, Zhang D, Jiang ZY et al (2016) Positive association between betatrophin and diabetic retinopathy risk in type 2 diabetes patients. Horm Metab Res 48(03):169-173

22. Wang H, Du L, Wu T et al (2017) Circulating betatrophin is associated with insulin resistance in humans: cross-sectional and interventional studies in vivo and in vitro. Oncotarget 8(57):96604-96614

23. Abu-Farha M, Cherian P, Qaddoumi MG et al (2018) Increased plasma and adipose tissue levels of ANGPTL8/betatrophin and ANGPTL4 in people with hypertension. Lipids Health Dis 17(35):1-23

24. Qu Q, Zhao D, Zhang F et al (2017) Serum betatrophin levels are increased and associated with insulin resistance in patients with polycystic ovary syndrome. J Int Med Res 45(1):193-202

25. Calan M, Yilmaz O, Kume T et al (2016) Elevated circulating levels of betatrophin are associated with polycystic ovary syndrome. Endocrine 53(1):271-279

26. Li L, Zhang F, Cui J et al (2016) Association of betatrophin with metabolic characteristics in overweight/obese and lean women with PCOS. Gynecol Endocrinol 33(3):238-243

27. Chen CC, Hendra S, Wen-Han C et al (2016) Higher serum betatrophin level in type 2 diabetes subjects is associated with urinary albumin excretion and renal function. Cardiovasc Diabetol 15(3): $1-24$

28. Siddiqa A, Cirillo E, Tareen SHK et al (2017) Visualizing the regulatory role of angiopoietin-like protein 8 (ANGPTL8) in glucose and lipid metabolic pathways. Genomics 109(5-6):408-418

Publisher's Note Springer Nature remains neutral with regard to jurisdictional claims in published maps and institutional affiliations. 